\title{
FUNCTION ALGEBRAS, MEANS, AND FIXED POINTS
}

\author{
BY \\ THEODORE MITCHELL( $\left.{ }^{1}\right)$
}

1. Introduction. A semigroup $S$ is said to have the common fixed point property on compacta if for each compact Hausdorff space $Y$, and for each homomorphic representation $\mathscr{S}$ of $S$ as a semigroup of continuous self-maps of $Y$, there is in $Y$ a common fixed point of $\mathscr{S}$. In [11, Theorem 1], it is shown that $S$ has the common fixed point property on compacta if and only if $m(S)$ has a multiplicative left invariant mean. A natural question is: does this result generalize to the case of a topological semigroup $S$ ?

It is shown in Corollary 1 that if $S$ is a topological semigroup such that $C(S)$ has a multiplicative Jeft invariant mean, then $S$ has the common fixed property on compacta with respect to continuous representations of $S$. But the proof of the converse encounters difficulties of the kind observed by M. Day in [3], namely that the family of adjoints of left translations on $C(S)$ fails to be a $w^{*}$-continuous representation on the desired subsets of $m(S)^{*}$. To remedy this, we employ $E$ representations of $S, C(S)$ on compact Hausdorff spaces; an analogue of the slightly continuous representations of $S$ that were introduced in [3].

In the main theorems of this paper, Theorems 1 and 2, it is shown that $C(S)$ has a multiplicative left invariant mean if and only if the pair $S, C(S)$ has the common fixed point property on compacta with respect to $E$-representations. In these theorems, additional implications are given concerning two other types of representations. In the proofs of Theorems 1 and 2, no use is made of the fact that the algebra $C(S)$ arises from a topology on $S$, so these results are stated in terms of closed subalgebras of $m(S)$.

Multiplicative left invariant means on $m(S)$ have been studied by the author in [11], and by E. Granirer in [6] and [7]. Subalgebras of $m(S)$ that have multiplicative left invariant means were first considered in [7].

Let $Y$ be a compact convex subset of a locally convex linear topological space, and let $S$ be a semigroup of continuous affine self-maps of $Y$ such that $m(S)$ has a left invariant mean. Day [2, Theorem 1] has shown that then $Y$ contains a common fixed point of $S$. Let $S^{\prime}$ be the convex hull (i.e., the set of finite convex combinations) of $S$. Since $S$ has a common fixed point in $Y$, then $S^{\prime}$ must also. In the light of our earlier remarks, it is tempting to conjecture that $m\left(S^{\prime}\right)$ has a multiplicative left invariant mean, but this conjecture does not hold. However, a subalgebra of $m\left(S^{\prime}\right)$ can be constructed which does have such a multiplicative mean. This algebra

Received by the editors November 25, 1966.

( ${ }^{1}$ ) This work was supported by National Science Foundation Grant GP-6327. 
could be obtained thus: let $y$ be a specific element of $Y$, let $\alpha: C(Y) \rightarrow m\left(S^{\prime}\right)$ be given by $(\alpha h) s^{\prime}=h\left(s^{\prime} y\right)$ for $h \in C(Y)$ and $s^{\prime} \in S^{\prime}$, then $\alpha(C(Y))$ can be shown to be an algebra with the desired properties. However, a somewhat more general approach than this is employed in $\S 5$, where the construction of the algebra is given.

2. Preliminaries and nomenclature. Let $S$ be a semigroup, $m(S)$ the space of all bounded real-valued functions on $S$, where $m(S)$ has the supremum norm. For $s \in S$, the left translation $l_{s}$ \{right translation $\left.r_{s}\right\}$ of $m(S)$ by $s$ is given by $\left(l_{s} f\right) s^{\prime}$ $=f\left(s s^{\prime}\right)\left\{\left(r_{s} f\right) s^{\prime}=f\left(s^{\prime} s\right)\right\}$, where $f \in m(S)$ and $s^{\prime} \in S$. Let $X$ be a subspace of $m(S)$, then $X$ is left $\{$ right $\}$ translation-invariant if $l_{s} X \subseteq X\left\{r_{s} X \subseteq X\right\}$ for all $s \in S$. If $X$ is both left and right translation-invariant, then $X$ is called translation-invariant.

Now let $X$ be a left translation-invariant closed subalgebra of $m(S)$ that contains $e$, the constant 1 function on $S$. An element $\mu \in X^{*}$ is a mean on $X$ if $\|\mu\|=1$ and $\mu(e)=1$. A mean $\mu$ on $X$ is left invariant if $\mu\left(l_{s} f\right)=\mu(f)$ for all $f \in X$ and $s \in S ; \mu$ is multiplicative if $\mu(f) \cdot \mu(g)=\mu(f \cdot g)$ (the pointwise product) for all $f, g \in X$.

When $Y$ is a topological space, $C(Y)$ denotes the space of all bounded realvalued continuous functions on $Y$, where $C(Y)$ has the supremum norm.

Let $S$ be a semigroup, $X$ a subset of $m(S)$, and $Y$ a compact Hausdorff space. Let $\eta$ be a homomorphism of $S$ onto $\mathscr{S}$, a semigroup (under functional composition) of continuous maps of $Y$ into itself. The set of all $y \in Y$ such that $T y(C(Y)) \subseteq X$ is denoted by $Y^{\prime}$, where the map $T y$ is given by $(T y h) s=h((\eta s) y)$, for $h \in C(Y)$, and $s \in S$. The family $\mathscr{S}$ is an E-representation of $S, X$ on $Y$ if $Y^{\prime}$ is nonempty, $\mathscr{S}$ is a $D$-representation if $Y^{\prime}$ is dense in $Y$, and $\mathscr{S}$ is an A-representation if $Y^{\prime}=Y$. (The symbol $E$ stands for exists, $D$ for dense, and $A$ for all.) The pair $S, X$ has the common fixed point property on compacta with respect to $i$-representations (where $i=E, D, A$; respectively) if, for each compact Hausdorff space $Y$, and for each $i$-representation of $S, X$ on $Y$, there is in $Y$ a common fixed point of the family $\mathscr{S}$.

\section{The main theorems.}

THEOREM 1. Let $S$ be a semigroup, $X$ a translation-invariant closed subalgebra of $m(S)$ that contains the constant functions. Then the following three conditions are equivalent:

(1) $X$ has a multiplicative left invariant mean.

(2) $S, X$ has the common fixed point property on compacta with respect to $E$ representations.

(3) $S, X$ has the common fixed point property on compacta with respect to $D$ representations.

Further, each of the equivalent conditions (1), (2), or (3) implies

(4) $S, X$ has the common fixed point property on compacta with respect to A-representations.

Proof. (1) $\rightarrow$ (2). Let $\eta$ be a homomorphism of $S$ onto $\mathscr{S}$, an $E$-representation of $S, X$ on the compact Hausdorff space $Y$. Then there exists an element $z \in Y$ 
such that $T z: C(Y) \rightarrow X$. Let $T z^{*}: X^{*} \rightarrow C(Y)^{*}$ be the adjoint map of $T z$, and let $\mu$ be a multiplicative left invariant mean on $X$. Then since $\mu$ is a mean,

$$
\left(T z^{*} \mu\right) 1=\mu(T z 1)=\mu(e)=1,
$$

where 1 designates the constant 1 function on $Y$. Also, a computation shows that $T z(h \cdot k)=T z h \cdot T z k$ (the pointwise product) for $h, k \in C(Y)$, so $T z^{*} \mu$ is a nonzero multiplicative linear functional on $C(Y)$ since $\mu$ is a multiplicative mean on $X$. But $Y$ is a compact Hausdorff space, so by [5, Lemma 25, p. 278], there exists an element $z^{\prime} \in Y$ such that

$$
h\left(z^{\prime}\right)=\left(T z^{*} \mu\right) h=\mu(T z h)
$$

for all $h \in C(Y)$. We will show that $z^{\prime}$ is the desired common fixed point of $\mathscr{S}$.

For each $s \in S$, define a map $\theta_{s}: C(Y) \rightarrow C(Y)$ by $\left(\theta_{s} h\right) y=h((\eta s) y)$ for $h \in C(Y)$, $y \in Y$. Then for each $s^{\prime} \in S$,

$$
\begin{aligned}
\left(T z\left(\theta_{s} h\right)\right) s^{\prime} & =\left(\theta_{s} h\right)\left(\left(\eta s^{\prime}\right) z\right)=h\left((\eta s)\left(\eta s^{\prime}\right) z\right) \\
& =h\left(\eta\left(s s^{\prime}\right) z\right)=(T z h)\left(s s^{\prime}\right)=\left(l_{s}(T z h)\right) s^{\prime}
\end{aligned}
$$

where the first and fourth equalities follow from the definition of $T z$, and the third follows from the fact that $\eta$ is a homomorphism. Therefore $\operatorname{Tz}\left(\theta_{s} h\right)=l_{s}(T z h)$. Thus it follows that for all $h \in C(Y)$ and $s \in S$,

$$
\begin{aligned}
h\left((\eta s) z^{\prime}\right) & =\left(\theta_{s} h\right) z^{\prime}=\mu\left(T z\left(\theta_{s} h\right)\right)=\mu\left(l_{s}(T z h)\right) \\
& =\mu(T z h)=h\left(z^{\prime}\right),
\end{aligned}
$$

where the fourth equality holds by virtue of the left invariance of $\mu$. But $C(Y)$ distinguishes between the points of $Y$ since $Y$ is a compact Hausdorff space, so $(\eta s) z^{\prime}=z^{\prime}$ for all $s \in S$.

(2) $\rightarrow$ (3). Condition (2) is formally stronger than (3).

$(3) \rightarrow(1)$. Let $Z \subseteq X^{*}$ be the set of all multiplicative means on $X$, and $Q$ the evaluation map $Q: S \rightarrow Z$ given by $(Q s) f=f(s)$ for $s \in S, f \in X$. Let $X^{*}$ have the $w^{*}$-topology, then $Z$ is a norm-bounded $\left(w^{*}\right.$-) closed subset of $X^{*}$. Choose $Y$ to be the closure of $Q(S)$ in $X^{*}$, then $Y$ is a compact Hausdorff space by [4, Corollary 3, p. 41], and $Q(S) \subseteq Y \subseteq Z \subseteq X^{*}$ where $Q(S)$ is dense in $Y$. For each $s \in S$, define a $\operatorname{map} L_{s}: X^{*} \rightarrow X^{*}$ by $L_{s}=l_{s}^{*}$. Each $L_{s}$ is $\left(w^{*}\right)$ continuous by [4, Theorem 2, p. 18], carries $Q(S)$ into $Q(S)$, and thus carries $Y$, the closure of $Q(S)$, into $Y$. Let $\mathscr{S}=\left\{L_{s} ; s \in S\right\}$, then $\mathscr{S}$ forms a semigroup, homomorphic to $S$, of continuous self-maps of $Y$. We wish to show that $\mathscr{S}$ is a $D$-representation of $S, X$ on $Y$.

The space $X$ is a closed subalgebra of $m(S)$ that contains $e$. By Kakutani's theorem on representations of abstract $(M)$-spaces (see [10, \$24.6, p. 242]), the elements of such a space $X$ evaluated on the space $Z$ yield an isometry of $X$ onto $C(Z)$. By the Tietze extension theorem [5, Theorem 3, p. 15], each function in $C(Y)$ is the restriction to $Y$ of some function in $C(Z)$; hence to each $h \in C(Y)$, 
there corresponds an $f_{h} \in X$ such that $h(\mu)=\mu\left(f_{h}\right)$ for $\mu \in Y$. (It can also be shown that $\|h\|=\left\|f_{h}\right\|$ and consequently that $Y=Z$, but this is not needed for the proof of Theorem 1.) Now suppose that $y \in Q(S)$, so $y=Q s^{\prime}$ for some $s^{\prime} \in S$. Then for $h \in C(Y)$ and $s \in S$,

$$
\begin{aligned}
(T y h) s & =h\left(L_{s} y\right)=h\left(l_{s}^{*}\left(Q s^{\prime}\right)\right)=\left(l_{s}^{*}\left(Q s^{\prime}\right)\right) f_{h} \\
& =\left(Q s^{\prime}\right)\left(l_{s} f_{h}\right)=\left(l_{s} f_{h}\right) s^{\prime}=f_{h}\left(s s^{\prime}\right)=\left(r_{s^{\prime}} f_{h}\right) s .
\end{aligned}
$$

Since $X$ is right translation-invariant, this gives us $T y h=r_{s^{\prime}} f_{h} \in X$; which means that $T y(C(Y)) \subseteq X$ if $y \in Q(S)$. But $Q(S)$ is dense in $Y$, thus $S$ is a $D$-representation of $S$, $X$ on $Y$. By (3), there exists $\mu_{0} \in Y$ such that $\mu_{0}=L_{s} \mu_{0}=l_{s}^{*} \mu_{0}$ for all $s \in S$, hence $\mu_{0}$ is the required multiplicative left invariant mean on $X$.

(3) $\rightarrow$ (4). Condition (3) is formally stronger than (4), which proves Theorem 1.

Suppose $S$ is a topological semigroup, that is, $S$ has a Hausdorff topology in which the semigroup product is continuous. It is known that $C(S)$ is a translationinvariant closed subalgebra of $m(S)$ that contains $e$, so Theorem 1 can be applied to the pair $S, C(S)$. Let $Y$ be a compact Hausdorff space, and $\eta$ an algebraic homomorphism of $S$ onto $\mathscr{S}$, a semigroup of continuous self-maps of $Y$. Then $\mathscr{S}$ is a continuous representation of $S$ on $Y$ if the map $S \times Y \rightarrow Y$ given by ( $\eta s$ ) $y$ for $s \in S$ and $y \in Y$, is continuous $\left({ }^{2}\right)$. The topological semigroup $S$ has the common fixed point property on compacta with respect to continuous representations if for each compact Hausdorff space $Y$, and each continuous representation of $S$ on $Y$, there is in $Y$ a common fixed point of $\mathscr{S}$.

Corollary 1. Let $S$ be a topological semigroup. If $C(S)$ has a multiplicative left invariant mean, then $S$ has the common fixed point property on compacta with respect to continuous representations.

Proof. Let $\mathscr{S}$ be a continuous representation of $S$ on a compact Hausdorff space $Y$. For $y \in Y$, the map $F y: S \rightarrow Y$ is continuous, where $F y$ is given by $F y s=(\eta s) y$ for $s \in S$. So for $y \in Y, h \in C(Y)$, and $s \in S$,

$$
(T y h) s=h((\eta s) y)=(h F y) s,
$$

thus $T y h \in C(S)$ since $h F y$ is the composition of two continuous functions. Therefore $\mathscr{S}$ is an $A$-representation of $S, C(S)$ on $Y$, hence Corollary 1 follows by Theorem 1.

The next corollary generalizes [11, Corollary 1] from the case of finite semigroups to that of compact topological semigroups. An element $s_{0}$ of a semigroup $S$ is a right zero of $S$ if $S s_{0}=\left\{s_{0}\right\}$.

$\left.{ }^{2}\right)$ Similarly, $\mathscr{S}$ is a separately continuous representation of $S$ on the compact Hausdorff space $Y$ if the map on $S \times Y$ is continuous in each variable. It can be shown that $\mathscr{S}$ is an $A$-representation of $S, C(S)$ on $Y$ if and only if $\mathscr{S}$ is a separately continuous representation of $S$ on $Y$. 
Corollary 2. Let $S$ be a compact semigroup. Then the following conditions are equivalent:

(1) $C(S)$ has a multiplicative left invariant mean.

(2) S has the common fixed point property on compacta with respect to continuous representations.

(3) $S$ has a right zero.

Proof. (1) $\rightarrow$ (2). This follows from Corollary 1 .

$(2) \rightarrow(3)$. The left multiplications of $S$ by elements $s \in S$ form a continuous representation of $S$ on itself. Since $S$ is compact, then by (2), $S$ has a right zero.

(3) $\rightarrow(1)$. If $s_{0}$ is a right zero of $S$, it follows by a routine computation that $Q s_{0}$ is a multiplicative left invariant mean on $C(S)$.

It was shown that condition (4) of Theorem 1 is implied by each of the other three equivalent conditions of the theorem. The converse implication was not shown; I do not know if it holds. However, the implication (3) $\rightarrow$ (1) of Theorem 1 was proven by use of the right translation-invariance of $X$. If to the hypotheses of the theorem, we add the requirement that $X$ satisfies a certain property that is stronger than right translation-invariance, then conditions (1)-(4) of Theorem 1 can be shown to be equivalent.

Let $X$ be a left invariant closed subspace of $m(S)$. For each $\mu \in X^{*}$, there is a $\mu_{l}: X \rightarrow m(S)$ given by $\left(\mu_{l} f\right) s=\mu\left(l_{s} f\right)$ for $f \in X$ and $s \in S$. The space $X$ is left introverted if $\mu_{l} X \subseteq X$ for all $\mu \in X^{*}$ (see Day [1, $\S 10$, p. 540]). Now let $X$ be a closed subalgebra of $m(S)$ which contains $e$. The algebra $X$ will be called left $M$ introverted if $\mu_{l} X \subseteq X$ for every multiplicative mean $\mu \in X^{*}$. It is easily verified that the statements (a) $X$ is left introverted, (b) $X$ is left $M$-introverted, and (c) $X$ is right translation-invariant, satisfy the relationship $(a) \rightarrow(b) \rightarrow(c)$.

THEOREM 2. Let $S$ be a semigroup, $X$ a left translation-invariant closed subalgebra of $m(S)$ that contains the constant functions. Let $X$, in addition, be left $M$-introverted. Then conditions (1)-(4) of Theorem 1 are equivalent.

Proof. Since $S, X$ satisfies the hypotheses of Theorem 1, we only need to show that (4) $\rightarrow(1)$. Choose the compact Hausdorff space $Y$ to be the set of all multiplicative means on $X$, where $Y$ is given the $w^{*}$-topology of $X^{*}$. Let $\mathscr{S}=\left\{L_{s} ; s \in S\right\}$, where $L_{s}=l_{s}^{*}$. By use of $[10, \S 24.6$, p. 242] again, it follows that for each $h \in C(Y)$, there exists a unique $f_{h} \in X$ such that $h(\mu)=\mu\left(f_{h}\right)$ for $\mu \in Y$. Then for $h \in C(Y)$, $\mu \in Y$ and $s \in S$, we have that

$$
\begin{aligned}
(T \mu h) s & =h\left(L_{s} \mu\right)=h\left(l_{s}^{*} \mu\right)=\left(l_{s}^{*} \mu\right) f_{h} \\
& =\mu\left(l_{s} f_{h}\right)=\left(\mu_{l} f_{h}\right) s .
\end{aligned}
$$

So $T \mu h=\mu_{l} f_{h} \in X$, since $X$ is left $M$-introverted; which means that $T y(C(Y)) \subseteq X$ for all $y \in Y$. Thus $\mathscr{S}$ is an $A$-representation of $S, X$ on $Y$. Since (4) asserts the pair $S, X$ has the common fixed point property on compacta with respect to $A$ representations, there exists $\mu_{0} \in Y$ such that $\mu_{0}=l_{s}^{*} \mu$ for all $s \in S$. 
4. Examples and remarks. (a) A modified version of Theorem 1 is obtained by dropping the requirement that $X$ is translation-invariant, and replacing it by the hypotheses that $X$ is left translation-invariant, and that the semigroup $S$ contains an identity $i$. Under these circumstances, it follows that conditions (1)-(4) of Theorem 1 satisfy the relationship:

$$
(1) \leftrightarrow(2) \rightarrow(3) \rightarrow(4) .
$$

The proof of $(1) \rightarrow(2) \rightarrow(3) \rightarrow(4)$ goes through as in Theorem 1; we will indicate the proof of $(2) \rightarrow(1)$. Let $Y$ be the space of multiplicative means on $X$, where $Y$ has the $w^{*}$-topology of $X^{*}$, and let $\mathscr{S}=\left\{l_{s}^{*} ; s \in S\right\}$. It can be verified that $Y^{\prime}$ is nonempty because $Q i \in Y^{\prime}$, so $\mathscr{S}$ is an $E$-representation of $S, X$ on $Y$, hence (1) follows.

(b) An "obvious proof" of the converse to Corollary 1 fails to go through. Let $Y$ be the space of multiplicative means on $C(S)$, where $Y$ is given the $w^{*}$ topology of $C(S)^{*}$, and let $\mathscr{S}=\left\{l_{s}^{*} ; s \in S\right\}$. If the topological semigroup $S$ has the common fixed point property on compacta with respect to continuous representations, and if it can be shown that $\mathscr{S}$ is a continuous representation of $S$ on $Y$, then it follows that $C(S)$ has a multiplicative left invariant mean. However, $\mathscr{S}$ need not be a continuous representation of $S$ on $Y$ even if $C(S)$ has, in fact, a multiplicative left invariant mean.

For a counterexample (cf. Day [3, Paragraph 1]), let $S$ be the semigroup of nonnegative real numbers under multiplication, where $S$ has the usual topology. Then $Q(0)$ can be verified to be a multiplicative left invariant mean on $C(S)$, so by Corollary 1, $S$ has the common fixed point property on compacta with respect to continuous representations. Let $\mu \in Y$ be a cluster point of $Q s$ as $s \rightarrow \infty$; such a point $\mu$ must exist by compactness of $Y$. Choose $f \in C(S)$ to be $f(s)=\min (s, 1)$ for $s \in S$. It is easily shown that $\left(l_{s}^{*} \mu\right) f=1$ if $s \neq 0$, and $\left(l_{s}^{*} \mu\right) f=0$ if $s=0$. Hence if $s \rightarrow 0$ in a deleted neighborhood of 0 , then $l_{s}^{*} \mu$ does not converge to $l_{0}^{*} \mu$, thus $\mathscr{S}$ is not a continuous representation of $S$ on $Y$. (Additionally, the family $\mathscr{S}$ serves as an example of a $D$-representation of $S, C(S)$ on $Y$ that is not also an $A$-representation.)

(c) In [11, Corollary 3], it was shown that if $S$ is a semigroup such that for every $s_{1}, s_{2} \in S$, there exists an $s_{3} \in S$ which satisfies $s_{1} s_{3}=s_{2} s_{3}$, then $m(S)$ has a multiplicative left invariant mean. The converse was obtained [11, Theorems 3 and 4] for the cases where $S$ is Abelian or has left cancellation; this converse was shown to hold for the general case by E. Granirer [6, Theorem 1].

(d) Suppose $X_{1}, X_{2}$ are left translation-invariant closed subalgebras of $m(S)$ that contain the constant functions, where $X_{1} \subseteq X_{2}$. If $X_{2}$ has a multiplicative left invariant mean, it can be shown that $X_{1}$ does also. This observation, together with (c) above, enables us to construct examples of pairs $S, X$ which have the common fixed point property on compacta with respect to the various representations. For an illustration, let $S$ be the real numbers with the usual metric topology, but with 
the semigroup product $s_{1} s_{2}=\max \left(s_{1}, s_{2}\right)$. Then by (c), $m(S)$ has a multiplicative left invariant mean, so $C(S)$ does also. By Corollary 1 , it follows that the topological semigroup $S$ has the common fixed point property on compacta with respect to continuous representations.

(e) Let $S$ be a semigroup. An example of an algebra $X \subseteq m(S)$ which has a multiplicative left invariant mean (even if $m(S)$ does not) is given by the trivial case where $X$ consists of all the constant functions on $S$.

(f) Another example of an algebra $X \subseteq m(S)$, where $m(S)$ does not have a multiplicative left invariant mean but $X$ does, is given by the case where $S$ is the positive integer under addition, and $X$ is the space of convergent functions on $S$. (For other examples, see Granirer [7].) Define $\mu \in X^{*}$ by $\mu(f)=\lim _{s \rightarrow \infty} f(s)$ for $f \in X$, then $\mu$ is a multiplicative left invariant mean on $X$. The pair $S, X$ can be shown to satisfy the hypotheses of Theorem 2 ; in particular, the space $X$ is left $M$-introverted.

(g) Now let $S$ be the group of integers under addition, and $X$ the space of all $f \in m(S)$ such that $\mu(f)$ exists, where $\mu(f)=\lim _{s \rightarrow+\infty} f(s)$. As in (f), $\mu$ is a multiplicative left invariant mean on $X$. But the pair $S, X$ now satisfies the hypotheses of Theorem 1, but not those of Theorem 2, since $X$ is not left $M$-introverted. In fact, it can be shown that there exists a multiplicative mean $\mu^{\prime} \in X^{*}$ such that $\mu_{l}^{\prime} X=m(S)$.

(h) Suppose $S$ is the group of integers under addition, but now let $X$ be the space of all $f \in m(S)$ such that both $\mu(f)$ and $\mu^{\prime}(f)$ exist, where $\mu(f)$ and $\mu^{\prime}(f)$ are the limits of $f(s)$ as $s \rightarrow+\infty$ and $s \rightarrow-\infty$, respectively. Then the pair $S, X$ satisfies the hypotheses of Theorem 2 , and $X$ has two distinct multiplicative left invariant means, $\mu$ and $\mu^{\prime}$.

(i) Let $S$ be a nontrivial regular Hausdorff space with the property that every continuous real-valued function on $S$ is constant; such a space exists by a result of E. Hewitt [9, Theorem 1, p. 503]. As in Granirer [8, p. 108], define a product on $S$ by the rule $s s^{\prime}=s$ for all $s, s^{\prime} \in S$. It is shown in [8] that $S$ is a topological semigroup. By remarks (c) and (e), $S$ provides an example of a topological semigroup for which $C(S)$ has a multiplicative left invariant mean, but $m(S)$ does not. By Corollary $1, S$ has the common fixed point property on compacta with respect to continuous representations. We can say even more than this, for by the proof of Corollary 1, if $\mathscr{S}$ is a continuous representation of $S$ on the compact Hausdorff space $Y$, then $\mathscr{S}$ is an $A$-representation of $S$ on $Y$. Thus $T y h \in C(S)$ for $y \in Y$ and $h \in C(Y)$, so $T y h$ is a constant function. Therefore $h((\eta s) y)=h\left(\left(\eta s^{\prime}\right) y\right)$ for $h \in C(Y), y \in Y$, and $s, s^{\prime} \in S$. But $C(Y)$ distinguishes between points of $Y$, so $\eta s=\eta s^{\prime}$ for all $s, s^{\prime} \in S$. Hence $\mathscr{S}$ is a singleton set $\mathscr{S}=\{\sigma\}$, where $\sigma$ is a retraction on $Y$ (recall $\mathscr{S}$ is a semigroup, so $\sigma$ is an idempotent). Thus $\sigma Y$ is the set of all common fixed points of $\mathscr{S}$.

5. Semigroups of finite means. Throughout this section, $S$ designates a semigroup and $X$ denotes a translation-invariant closed subspace of $m(S)$ that contains 
the constant functions. It will be shown that a new pair $P, W$ can be constructed from $S, X$ such that $W$ has a multiplicative left invariant mean if and only if $X$ has a left invariant mean. This construction furnishes a source of examples of pairs $P, W$ for which $W$ has a multiplicative left invariant mean, but for which $m(P)$ does not.

Let $P$ be the set of all real-valued functions $p$ on $S$ that satisfy (1) $p(s) \geqq 0$ for all $s \in S$, (2) $p(s)>0$ for at most a finite number of elements of $S$, and (3) $\sum_{s \in S} p(s)=1$. Define a product on $P$ by

$$
p p^{\prime}(s)=\sum_{a b=s} p(a) p^{\prime}(b)
$$

for $p, p^{\prime} \in P$ and $a, b, s \in S$. In other words, $P$ is the semigroup of finite means on $S$ with the convolution operation of $l^{1}(S)$ as a product. Let $\tau: X \rightarrow m(P)$ be given by

$$
(\tau f) p=\sum_{s \in S} p(s) f(s)
$$

for $f \in X$ and $p \in P$. Then the closed subalgebra of $m(P)$ that is generated by $\tau X$ is denoted by $W$, i.e., $W$ is the smallest closed subalgebra of $m(P)$ that contains $\tau X$. Let $I: S \rightarrow P$ be the map where $I s$ is the characteristic function of $s$.

We list some useful facts about $P$ and $W$; see Day $[1, \S 5]$ for some of these items.

$$
\begin{aligned}
I\left(s s^{\prime}\right) & =(I s)\left(I s^{\prime}\right) \text { for } s, s^{\prime} \in S . \\
p & =\sum_{s \in S} p(s) I s \text { for } p \in P . \\
p p^{\prime} & =\sum_{a \in S} \sum_{b \in S} p(a) p^{\prime}(b) I(a b) \text { for } p, p^{\prime} \in P . \\
(\tau f)(I s) & =f(s) \text { for } f \in X \text { and } s \in S . \\
\tau e & =e^{\prime}, \text { the constant } 1 \text { function on } P . \\
\|\tau\| & =1 .
\end{aligned}
$$

LEMMA 1. The algebra $W$ is translation-invariant.

Proof. This is shown only for left translation-invariance; the proof is similar for right translation-invariance. For $f \in X$ and $p, p^{\prime} \in P$, it follows that

$$
\begin{aligned}
\left(l_{p} \tau f\right) p^{\prime} & =(\tau f)\left(p p^{\prime}\right)=(\tau f)\left(\sum_{a \in S} \sum_{b \in S} p(a) p^{\prime}(b) I(a b)\right) \\
& =\sum_{a \in S} \sum_{b \in S} p(a) p(b) f(a b)=\sum_{a \in S} p(a) \sum_{b \in S} p^{\prime}(b)\left(\left(l_{a} f\right) b\right) \\
& =\sum_{a \in S} p(a)\left(\left(\tau l_{a} f\right) p^{\prime}\right)=\left(\sum_{a \in S} p(a) \tau l_{a} f\right) p^{\prime} .
\end{aligned}
$$

Thus we have

$$
l_{p} \tau f=\sum_{a \in S} p(a) \tau l_{a} f=\tau\left(\sum_{a \in S} p(a) l_{a} f\right) .
$$


But $l_{a} f \in X$ since $X$ is left translation-invariant, so $l_{p} \tau f \in W$ for $f \in X$ and $p \in P$. This means that $\tau X$ is left translation-invariant. However, $l_{p}$ is a bounded multiplicative linear operator on $m(P)$, hence the Banach algebra $W$ generated by $\tau X$ is also left translation-invariant.

Since $W$ is a translation-invariant closed subalgebra of $m(P)$ that contains the constant functions, it is meaningful to speak of a multiplicative left invariant mean on $W$.

THEOREM 3. The algebra $W$ has a multiplicative left invariant mean if and only if $X$ has a left invariant mean.

Proof. From equations (5) and (6), it follows that if $\mu$ is a mean on $W$, then $\tau^{*} \mu$ is a mean on $X$. For $f \in X, s \in S$, we have

$$
l_{I s} \tau f=\tau\left(\sum_{a \in S}((I s) a) l_{a} f\right)=\tau l_{s} f
$$

by equation (7). Let $\mu$ be a left invariant mean on $W$, then

$$
\left(\tau^{*} \mu\right)\left(l_{s} f\right)=\mu\left(\tau l_{s} f\right)=\mu\left(l_{I s} \tau f\right)=\mu(\tau f)=\left(\tau^{*} \mu\right) f
$$

for all $f \in X$ and all $s \in S$. Hence $\tau^{*} \mu$ is a left invariant mean on $X$, which shows the "only if" implication.

For the converse, let $\lambda$ be a left invariant mean on $X$. Let $q: P \rightarrow X^{*}$ be the map given by

$$
(q p) f=\sum_{s \in S} p(s) f(s)=(\tau f) p,
$$

where $p \in P$ and $f \in X$. Then $q P$ is $w^{*}$-dense in the set of means on $X$ since $P$ is the set of finite means on $S$ (see $\left[1, \S 10\right.$, p. 540]), so there exists a net $\left\{q p_{\gamma}\right\}$ which is $w^{*}$-convergent to $\lambda$. Let $Y$ be the set of all multiplicative means on $W$, and let $Q: P \rightarrow Y$ be the evaluation map $(Q p) h=h(p)$ for $p \in P$ and $h \in W$. By compactness of $Y$ in the $w^{*}$-topology of $X^{*}$, the net $\left\{Q p_{\gamma}\right\}$ has a subnet $\left\{Q p_{\delta}\right\}$ that converges $w^{*}$ to some $\mu \in Y$. We will show that $\mu$ is the desired multiplicative left invariant mean on $W$.

For $p_{\delta} \in\left\{p_{\delta}\right\}$ and $f \in X$, we have

$$
\left(\tau^{*} Q p_{\delta}\right) f=\left(Q p_{\delta}\right)(\tau f)=(\tau f) p_{\delta}=\left(q p_{\delta}\right) f
$$

hence $\tau^{*} Q p_{\delta}=q p_{\delta}$. But $\left\{Q p_{\delta}\right\}$ and $\left\{q p_{\delta}\right\}$ converge $w^{*}$ to $\mu$ and $\lambda$ respectively, so $\tau^{*} \mu=\lambda$ by $w^{*}$-continuity of $\tau^{*}$. If $p \in P$ and $f \in X$, we obtain

$$
\begin{aligned}
\mu\left(l_{p} \tau f\right) & =\mu\left(\sum_{a \in S} p(a) \tau l_{a} f\right)=\sum_{a \in S} p(a)\left(\left(\tau^{*} \mu\right) l_{a} f\right) \\
& =\sum_{a \in S} p(a) \lambda\left(l_{a} f\right)=\sum_{a \in S} p(a) \lambda(f) \\
& =\lambda(f)=\left(\tau^{*} \mu\right) f=\mu(\tau f),
\end{aligned}
$$

where the first equality follows from equation (7), and the fourth from the left invariance of $\lambda$. This means that $\mu$ is left invariant when restricted to $\tau X$. But $\mu$ 
and $l_{p}$ are multiplicative, continuous, and linear; hence $\mu$ is left invariant on $W$, the Banach algebra generated by $\tau X$, which proves Theorem 3 .

It can also be shown that $\tau^{*}$ is a $w^{*}-w^{*}$ homeomorphism of $Y$ onto the set of means of $X$, but this is not needed for the proof above.

By use of Theorem 3, examples can be obtained of pairs $P, W$ which have a multiplicative left invariant mean, and so by Theorem 1, have the common fixed point property on compacta with respect to $E, D$, and $A$-representations. Any topological semigroup $S$ for which $C(S)$ has a left invariant mean will serve admirably as a source of a suitable pair $S, X$ if $X$ is taken to be $C(S)$. Some wellknown examples are Abelian topological semigroups, and compact topological groups. Another possibility is the use of appropriate subspaces of $C(S)$; for example, if $S$ is a topological group, then $X$ can be taken to be the space of almost periodic functions on $S$.

If $S$ is a semigroup such that $m(S)$ has a left invariant mean, then the space $m(P)$ need not have a multiplicative left invariant mean. To illustrate this, let $S$ be the group of integers under addition, and let $X=m(S)$. A computation shows that $P$ is an infinite Abelian cancellation semigroup. But if $m\left(S^{\prime}\right)$ has a multiplicative left invariant mean, where $S^{\prime}$ is a cancellation semigroup, then $S^{\prime}$ is a singleton set by [11, Theorem 2]; hence $m(P)$ does not have a multiplicative left invariant mean. It follows that $W$ must be a proper subset of $m(P)$, since $W$ does have such a mean by Theorem 3 .

\section{BIBLIOGRAPHY}

1. M. M. Day, Amenable semigroups, Illinois J. Math. 1 (1957), 509-544.

2. - Fixed-point theorems for compact convex sets, Illinois J. Math. 5 (1961), 585-590.

3. - Correction to my paper "Fixed-point theorems for compact convex sets," Illinois J. Math. 8 (1964), 713.

4. —_ Normed linear spaces, 2nd ed., Ergebnisse der Mathematik und ihrer Grenzgebiete, Springer, Berlin, 1962.

5. N. Dunford and J. T. Schwartz, Linear operators, Part I, Pure and Appl. Math. No. 7, Interscience, New York, 1958.

6. E. Granirer, Extremely amenable semigroups, Math. Scand. 17 (1965), 177-197.

7. —- Extremely amenable semigroups. II, Math. Scand. (to appear).

8. - On the invariant mean on topological semigroups and on topological groups, Pacific J. Math. 15 (1965), 107-140.

9. E. Hewitt, On two problems of Urisohn, Ann. of Math. (2) 47 (1946), 503-509.

10. J. L. Kelley and I. Namioka (et al.), Linear topological spaces, Van Nostrand, Princeton, N. J., 1963.

11. T. Mitchell, Fixed points and multiplicative left invariant means, Trans. Amer. Math. Soc. 122 (1966), 195-202.

State University of New York at Buffalo, Buffalo, NeW YorK 